\title{
La Distomatose hépatique en Algérie
}

\author{
Par Alexis COUMBARAS
}

La distomatose hépatique a été peu étudiée en Algérie. La maigre bibliographie, que nous analysons brièvement, à la fin de ce travail, constitue, sauf omission, une liste complète de tout ce qui a été publié à ce jour.

Tout porte à croire cependant que cette maladie y est bien plus fréquente que ne le laisse supposer le petit nombre de cas publiés. Il faut se souvenir qu'il y a dix ou vingt ans à peine, la distomatose était considérée comme une maladie exceptionnelle en Europe. Elle ne l'était certainement pas, mais rares étaient les cas correctement diagnostiqués. Il en fut certainement de même, à cette époque, en Algérie.

Dans une deuxième période, le perfectionnement des techniques coprologiques, l'introduction des méthodes immunologiques rendirent le diagnostic plus facile, et un certain regain d'intérêt pour une maladie, qui fut pendant longtemps considérée comme une rareté, fit que les publications se multiplièrent: communications de cas isolés, descriptions purement cliniques d'abord, études épidémiologiques, de plus en plus complètes, ensuite.

En Algérie, la connaissance de la distomatose ne fit pas de tels progrès : la situation politique et militaire, qui y a régné ces derniers temps, et la détérioration de l'infrastructure sanitaire et médicale, qui s'en est suivie, n'étaient pas propices à ce genre d'investigations, et encore moins à des travaux épidémiologiques sur le terrain. Mais nous verrons que, si ces raisons de conjoncture sont en grande partie responsables de ce que cette maladie est peu connue en Algérie, il existe aussi des raisons purement épidémiologiques qui en font, dans ce pays, une maladie plus rare qu'ailleurs.

En été 1963, il nous a été donné d'observer, dans la région de Bougie, une épidémie de distomatose hépatique, survenue dans une petite communauté européenne, intéressant douze personnes en tout. Certaines particularités cliniques et les résultats de l'étude épidémiologique entreprise à cette occasion nous ont paru utiles d'être rapportés. Voici les faits :

\section{Aspects cliniques}

Le 15 juin 1963, M. Dom., 30 ans, vient nous consulter pour un ensemble de troubles mal définis se résumant en quelques intolérances alimentaires, des nausées fréquentes, des douleurs sourdes dans la région hépatique, un peu de fièvre, de temps en temps. Il y a 
un mois, il aurait fait un ictère avec fièvre, mais qui n'aurait duré que trois jours. Mais surtout, M. Dom. se sent très fatigué. Il a maigri de $8 \mathrm{~kg}$ en deux mois et ne peut plus travailler. Il avait bénéficié, à ce jour, de toutes sortes de traitements : successivement, il avait reçu de la nivaquine, des antibiotiques variés, de la vitamine B12, sans aucun résultat. Les antispasmodiques diminuent la douleur à l'hypochondre droit et les phénomènes nauséeux, mais de manière incomplète et transitoire.

A l'examen, l'état général paraît conservé. Le foie ne déborde pas le rebord costal, mais la percussion un peu énergique de la région hépatique est nettement douloureuse. C'est l'hémogramme qui nous fait suspecter la distomatose hépatique : leucocytose à 22.000, avec $57 \%$ d'éosinophiles. L'examen parasitologique des selles, pratiqué après concentration, selon la technique de Telemann, est négatif à deux reprises; négative également la recherche des œufs de douve dans la bile, après tubage. Mais l'intradermoréaction à l'antigène distomien de l'Institut Pasteur confirme ce diagnostic : formation, en quelques minutes, d'un gros placard ortié, et, quelques heures plus tard, d'un œdème impressionnant, intéressant tout l'avant-bras, doublant le volume de celui-ci. Edème blanc, non douloureux, gardant le godet, et mettant trois jours pour se résorber. Les intradermoréactions, pratiquées sur l'autre avant-bras, à titre de témoin, avec de l'eau physiologique, de l'antigène ascaridien et de l'antigène hydatique, sont négatifs.

Par ailleurs, interrogé à ce sujet, le malade se souvient avoir mangé, il y a deux mois environ, du cresson que lui avait vendu un colporteur.

Nous avions ainsi réuni un ensemble d'arguments suffisant en faveur de la distomatose hépatique pour entreprendre le traitement, bien que nous n'ayons pas trouvé les œufs de douve, trop rares peut-être, pour être mis en évidence par les seules techniques classiques dont nous disposions, ou même absents, étant donné le caractère relativement récent de l'infestation.

Il semble qu'à l'heure actuelle, c'est la déhydro-émétine qui constitue le meilleur traitement de la distomatose hépatique. Aussi, nous lui avons administré ce produit, à raison de $1 \mathrm{mg}$ par kilo de poids corporel et par jour, en injections sous-cutanées, dans la région interscapulo-vertébra.e, pendant dix jours.

Ce traitement a été bien supporté, le malade ayant été mis au repos, sans toutefois avoir été hospitalisé. Nous n'avons noté aucun signe d'intoxication émétinienne. Par contre, dès le milieu de la cure, les douleurs dans la région hépatique régressent, puis disparaissent, et l'état général s'améliore sensiblement. La numération des globules blancs revient à la normale, l'éosinophilie tombe à $8 \%$, et le malade reprend son activité professionnelle quinze jours après la fin de son traitement.

Jusque-là, rien que de très banal. Mais c'est ici que se place un incident, qui mérite d'être rapporté.

La très forte positivité de l'intradermo-réaction à l'antigène distomien, qui avait permis de faire le diagnostic, nous avait frappé, et nous avions voulu voir comment réagirait le sujet à une deuxième intradermo-réaction, pratiquée après cette guérison apparente, escomptant une diminution de l'intensité de la réaction.

Or, dans les quelques minutes qui ont suivi l'injection strictement intradermique 
de $1 / 10^{\circ}$ de $\mathrm{ml}$. d'antigène, notre malade se sent mal, s'effondre sur un lit et a juste le temps de déclarer qu'il étouffe, avant de perdre connaissance. Sa face est d'une pâleur livide, les lèvres exsangues, les yeux révulsés, tout le corps est parcouru d'un fin tremblement. La tension artérielle tombe à $8 / 5$, le pouls est à $120 / \mathrm{mn}$, la respiration est sifflante, superficielle, difficile, mais l'auscultation des poumons ne révèle rien d'anormal.

Nous transportons le malade sous une tente à oxygène et nous lui injectons, par voie intraveineuse, un mélange de phénergan-hydrocortisone, ainsi que de la coramine en sous-cutanée.

Dix minutes après, le malade recouvre sa conscience, et tout rentre dans l'ordre. Il ne subsiste, de cet incident impressionnant mais fugace, qu'une grande lassitude et une intradermo-réaction encore une fois très positive à l'avant-bras. L'œdème s'étendra à tout l'avant-bras dans les heures qui suivent, aussi important que la première fois, et, comme la première fois, mettra trois jours à disparaître.

Nous ne pensons pas qu'a priori on pouvait prévoir cet incident. Mais, après coup, il nous semble aisément explicable par un mécanisme anaphy!actique. Chez ce sujet, déjà fortement sensibilisé, comme le prouve une première intradermo-réaction très fortement positive, la lyse, par le médicament d'un certain nombre de douves, encore enchâssées en plein tissu hépatique, a provoqué une hausse importante d'anticorps. La deuxième intradermo-réaction, avec son apport d'antigènes, si minime soit-il, a joué le rôle d'une injection de rappel et a déclenché une réaction allergique violente. Il eût été intéressant de rechercher in vitro, par des réactions immunologiques quantitatives, cette richesse en anticorps du sérum du malade, avant et après le traitement. Malheureusement, nous n'étions pas en mesure de le faire. Nous pensons néanmoins qu'il était utile de signaler cet incident, pour attirer l'attention sur le danger qu'il peut y avoir à pratiquer une intradermo-réaction après le traitement d'une distomatose hépatique, surtout si une première intradermo-réaction s'était montrée fortement positive, traduisant un état fortement allergique.

Si l'incident, qui vient d'être décrit, n'eut pas de suites, notre malade n'en fut pas, pour autant, au bout de ses peines. En effet, tout nous portait à croire à une guérison définitive quand, un mois et demi environ après la fin du traitement, la symptomatologie de la distomatose réapparut, et l'éosinophilie remontait à $14 \%$. Devant cette rechute, nous prescrivons une deuxième cure de déhydro-émétine, identique à la précédente. Ce traitement fut moins bien supporté : asthénie, dérobement des jambes, quelques palpitations sans tachycardie vers la fin de la cure, qui a pu néanmoins être suivie complètement, et qui fut définitive dans ses résultats. Régulièrement revu depuis, pendant un an, M. Dom. ne se plaint plus de rien ; il gardera toutefois, un an après la deuxième cure, une éosinophilie résiduelle à $5 \%$.

Dès que le diagnostic de distomatose hépatique fut soupçonné, nous avons procédé à une enquête dans l'entourage de notre malade. On connaît, en effet, le caractère familial de la maladie, dû au fait que c'est à la table familiale que se fait l'infestation. La maladie peut aussi présenter un caractère franchement épidémique. Elle s'étend d'autant plus que le cresson infestant connaît une diffusion et un succès plus grands au sein de la population considérée. 
M. Dom. est instituteur dans une petite localité appelée Les Falaises, située entre Bougie et Ziama-Mansouriah, à $7 \mathrm{~km}$ de Ziama-Mansouriah, sur la route côtière qui va de Bougie à Djidjelli. Ce petit village algérien ne comporte que quatre familles françaises d'origine métropolitaine. Ces familles y sont établies depuis peu, venues servir en Algérie au titre de la coopération culturelle et technique franco-algérienne. Elles forment une petite communauté très homogène et très liée, partageant souvent leurs loisirs et leurs repas, ayant les mêmes habitudes alimentaires et le même approvisionnement en cresson sauvage, fourni par le même colporteur, qui le cueille toujours au même endroit. Aussi, notre enquête, qui débuta par cette communauté, fut facile. Voici ce que nous avons trouvé, le tout brièvement exposé, car les cas de distomatose hépatique ainsi dépistés furent banaux quant à leur expression clinique et biologique, et quant à leur évolution, également banale, vers la guérison, sous l'effet du traitement de la déhydro-émétine.

M. Boi., sans parenté avec les précédents, souffre depuis un mois de douleurs dans la région hépatique, qu'il traite par des antispasmodiques et des cholagogues, sans grand résultat. Leucocytes : 35.800 . Eosinophilie à $66 \%$. Intradermo-réaction : $+^{+}+$. Recherche des œufs de Fasciola hepatica dans les selles et la bile, méthode Telemann: négative. Malade traité et guéri par dix injections quotidiennes de déhydro-émétine, à raison de $1 \mathrm{mg}$ par kilo de poids corporel et par jour. Traitement bien supporté. Guérison définitive après une seule cure.

M. P. (Fr.) et sa femme, Mme P. (J.), sans liens de parenté avec les précédents, viennent consulter spontanément. Une partie de leur famille, six personnes en tout, qui ont quitté Les Falaises il y avait un mois de cela, pour raisons de service, se trouvent hospitalisées à Trêves, en Allemagne Fédérale, pour distomatose hépatique. (Ces malades font l'objet d'une publication par M. Fromantin, P. Riberi, Ch. Rossazza, Carnus, cf. bibliographie). Eux-mêmes ne se sentent pas bien depuis quelque temps.

M. P. (Fr.) se plaint de fatigue, d'amaigrissement, de quelques douleurs abdominales diffuses, de quelques nausées. Mais tous ces troubles sont, dans l'ensemble, bien supportés. Le malade les attribue au surmenage et à des troubles digestifs banaux. Leucocytes : 26.500, éosinophilie à $63 \%$. Intradermo-réaction : ++ . Recherche des œufs de $F$. hepatica dans les selles et la bile, méthode Telemann : négative.

Mme P. (J.) ne se plaint que de nausées, qu'elle attribuait jusqu'alors à une grossesse en cours d'évolution. Leucocytes: 14.200, éosinophilie à $11 \%$. Intradermo-réaction: ++ . Recherche des œufs de $F$. hepatica dans les selles: négative.

Enfant P. (J.-Cl.), 6 ans, ne se plaint de rien. Mais: leucocytes : 10.000, éosinophilie à $11 \%$. Intradermo-réaction faiblement positive. Recherche des œufs de $F$. hepatica dans les selles : négative.

Enfant P. (C.), 7 ans, ne se plaint de rien. Leucocytes : 9.000, éosinophilie : $6 \%$. Intradermo-réaction : négative. Recherche des œufs de $F$. hepatica dans les selles : négative. Notre opinion fut, pendant quelque temps, réservée au sujet de cet enfant. En fait, nous avons attendu vainement le virage de l'intradermo-réaction, et l'éosinophilie est tombée à $1 \%$, après traitement de l'oxyurose.

M. Dor., père de Mme P., se dit parfaitement bien portant. Leucocytes: 6.000 , mais éosinophilie à $18 \%$, et intradermo-réaction : + . Recherche des œufs de $F$. hepatica dans les selles : négative. 
Tous ces malades ont bénéficié du traitement à la déhydro-émétine, selon le schéma précédemment décrit. Ils l'ont tous très bien supporté. M. et Mme P. ont suivi deux cures de déhydro-émétine, la première cure n'abaissant l'éosinophilie, respectivement, qu'à 11 et $6 \%$, la deuxième cure amenant la guérison définitive, clinique et biologique : éosinophilie à $2 \%$, après un recul de six mois.

Leur fils P. (J.-Cl.) fut guéri définitivement, par une seule cure.

M. Dol. a profité de son congé en Allemagne pour se faire traiter à Trêves. Même schéma thérapeutique. Guérison avec une seule cure. Quelques signes d'intoxication émétinienne sans gravité. Les autres malades soignés en Allemagne furent également guéris par une ou deux cures de déhydro-émétine, suivant le même schéma thérapeutique.

\section{Aspects épidémiologiques}

Nous avons recherché et retrouvé le colporteur de cresson sauvage, qui nous indiqua le lieu de la cueillette. Il se trouve sur la route de Bougie à Sétif, à $4 \mathrm{~km} \mathrm{du}$ croisement Bougie-Djidjelli, Bougie-Sétif.

Là, en contrebas de la route qui s'accroche au flanc de la montagne, sur un sol très humide, parcouru de nombreux ruissellements, qui accompagnent le cours de l'oued principal, pousse une cressonnière très touffue. Le débit de l'oued est très irrégulier au cours de l'année, comme c'est la règle en Afrique du Nord. Il en est de même du débit des sources, nombreuses à cet endroit, et des ruissellements adventices, mais il subsiste, tout au long de l'année, même pendant les périodes de sécheresse, suffisamment d'humidité pour assurer la pérennité de la cressonnière.

De nombreux troupeaux de vaches et de moutons paissent dans cette vallée, où ils trouvent un très riche pâturage. L'inspecteur-vétérinaire de Bougie, consulté à ce sujet, nous a déclaré que, bien qu'il ne tenait pas une statistique précise à ce sujet, il estimait à $60 \%$ environ la proportion des bovins et ovins atteints de distomatose hépatique à Fasciola hepatica dans cette région.

Nous avons également trouvé quelques limnées sur les plants de cresson. Les mollusques étaient peu abondants et les dix-sept spécimens, que nous avons disséqués, ne présentaient pas de signes d'un parasitisme quelconque: nous n'avons trouvé ni cercaires, ni sporocystes. Evidemment, le nombre de mollusques examinés est tout à fait insuffisant ; de plus, la période de l'année où a été menée cette partie de l'enquête (mois de décembre) était inadéquate : mollusques rares, cycle parasitaire allongé à basse température. Par contre, dans des échantillons de selles prélevées sur des bouses de vache fraîchement émises, nous avons trouvé des œufs de $F$. hepatica tout à fait caractéristiques, dans quatre cas sur dix, rien qu'à l'examen direct.

Toutes les conditions étaient donc réunies, aux Falaises, pour permettre l'éclosion de cette épidémie de douze personnes. Bien plus, il devenait étonnant de voir l'épidémie en rester là, de la voir se limiter à cette petite communauté de Français. En fait, ici, comme dans bien d'autres maladies parasitaires, ce sont les habitudes alimentaires qui expliquent cette limitation à la seule communauté européenne de la distomatose hépa- 
tique. Nous nous en sommes rendus compte dès que nous avons voulu étendre notre enquête à la population algérienne de la région. Celle-ci ignore totalement le cresson en tant que salade comestible et n'en consomme jamais. Quelques rares familles rurales connaissent le cresson, mais ne le consomment que cuit, et par conséquent en toute sécurité. Si on admet que ces habitudes alimentaires sont les mêmes dans toute l'Algérie, voilà qui explique que toute la bibliographie de la distomatose hépatique en Algérie ne porte que sur des cas survenus chez des Européens. Il en est certainement de même au Maroc, où la bibliographie, beaucoup plus abondante sur la distomatose hépatique, porte sur plus de cent cas, et où on ne relève, dans une publication de M. Malenfant, que quelques cas de distomatose chez des musulmans marocains. Encore s'agit-il de cas ayant éclaté dans une petite équipe d'ouvriers français et marocains, vivant momentanément en communauté dans une région, où ils ont été transportés pour des raisons professionnelles. Il s'agit donc d'un cas d'espèce où, à la faveur d'une vie communautaire étroite, les ouvriers marócains ont adopté le régime alimentaire des Français, et ont mangé, comme eux, du cresson cru en salade.

La non consommation de cresson par la très grande majorité de la population de l'Algérie fait que cette denrée n'est pas régulièrement commercialisée sur les marchés algériens, malgré l'abondance des cressonnières sauvages. Cela constitue, dans une certaine mesure, une protection pour la population européenne des villes. Nous en avons vu cependant, rarement il est vrai, sur le marché de Bougie. Les infestations se feront donc, avant tout, à la faveur d'une cueillette individuelle par le consommateur lui-même, ou encore par l'entremise d'un colportage occasionnel. Cela explique aussi, à côté de raisons déjà évoquées, la relative rareté des cas de distomatose hépatique, signalés en Algérie. Dans tous les cas, il s'agit de cresson sauvage, poussant dans des sites naturels, souvent très fréquentés par des troupeaux d'ovins et de bovins. Dans les meilleurs cas, il s'agit de plants de cresson poussant dans les vergers, plus ou moins spontanément. Là, ils ne sont en général tolérés par l'exploitant qu'en dehors des surfaces utiles, c'est-à-dire, avant tout, le long des palissades, plus ou moins précaires. Celles-ci ne constituent qu'une barrière tout à fait illusoire contre l'infestation par les eaux courantes, souillées par les excréments des animaux qui passent à proximité.

Tous les éléments nécessaires à l'expansion de la distomatose hépatique sont donc réunis en Algérie, mais les habitudes alimentaires de la majeure partie de la population la limitent au seul élément européen. C'est certainement, à côté des raisons évoquées plus haut, la cause de la relative rareté de cette maladie en Algérie.

\section{Résumé}

Etude d'une épidémie de distomatose hépatique en Algérie, touchant douze personnes. Excellents résultats du traitement par la déhydro-émétine, dans tous ces cas de distomatose traitée précocément. Choc anaphylactique observé à la suite d'une intradermo-réaction à l'antigène distomien, pratiquée après un traitement. Aspects épidémiologiques de la distomatose en Algérie. Maladie strictement réservée à l'élément européen de la population, les Algériens ne consommant pas de cresson à l'état cru. 


\section{Bibliographie}

Vital (A.), 1845. - Description du premier cas de distomatose hépatique en Algérie (Constantinois). Découverte d'autopsie. Gazette médicale, 3, 274.

Dionis des CARrières, 1857 (cité par Davaine en 1877). - Un cas de localisation erratique sous-cutanée (région de Constantine). Traité des Entozoaires, Paris, 2édition, p. 386.

MaURY et PéLissier, 1924. - Un cas d'obstruction du cholédoque par une grande douve à Médéa. Presse médicale, $\mathrm{n}^{\circ} 20$.

Desage, 1926. - Un cas de distomatose hépatique à Aïn-Khial (Oranie). Bull. Mém. Soc. Méd. Hôp. Paris, 30 avril.

Sénevet (G.) et Champagne (R.), 1929. - Un cas de distomatose hépatique à Sétif. Arch. Inst. Pasteur d'Algérie, 7, 207-216.

Delanó́ (P.), 1929. - Un cas de distomatose hépatique à Blida. Bull. Soc. Path. Exot., 8, 673-674.

NouchI, 1929. - Découverte foriuite d'œufs de $F$. hepatica dans la bile d'un malade atteint de giardiose vésiculaire. Certainement œufs en transit. Thèse Alger.

Chatron (M.), 1935. - Un cas de distomatose hépatique chez un jeune homme ayant fait son service militaire en Algérie. Bull. Soc. Path. Exot., 26, 24-25.

Monceaux (A.) et Alcay (M.), 1939. - Un cas de distomatose hépatique à Alger. Bull. Soc. Path. Exot., 32, 169-172.

FrIESS (M.), 1946. - Infestation familiale à $F$. hepatica à Sétif: trois malades. Algérie médicale, mai-juin, 247-253.

JACQUemin (P.), Rool (G.), 1957. - Un cas de dis.omatose hépatique chez un malade ayant séjourné à Blida et à Alger. Algérie médicale, septembre, 61, 9, 1011-1021.

BÉrÉNI (J.) et Duboureau (L.-H.), 1963. - Un cas de distomatose hépatique contractée à Perregaux (Oranie), traité à la déhydro-émétine. Soc. Médecine Milit. Française, 8 , 317-320.

Fromantin (M.), Ribéri (P.), Rossazza (Ch.), Carnus, 1963. - Remarques sur les critères de guérison de la distomatose hépatique, traitée par la déhydro-émétine. A propos d'une épidémie familiale survenue dans la région de Bougie. Soc. Médecine Milit. Française, 10, 425-430.

Malenfant (M.), 1961. - Maroc médical, 40, 1244-1299.

(Institut de Parasitologie [Directeur: $\mathrm{P}^{\mathrm{r}}$ L.-Ch. BRUMPT].

Faculté de Médecine de Paris) 